\title{
Professionalism in Peer Review
}

\author{
Harry J. Levinson \\ Editor-in-Chief
}

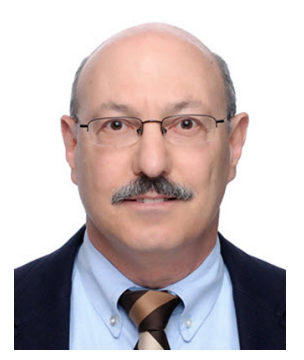

Peer review is an important element of the editorial process for ensuring the quality of papers published in scientific and technical literature. $\mathrm{JM}^{3}$ uses a peer review process similar to many other journals: the process is single blind, meaning the reviewer knows the author's name, but the author does not know the reviewer's name; and each manuscript is assigned to at least two people for review. Reviewers are technologists and scientists who are recognized by members of the journal's editorial board as individuals who have expertise in the subject matter of the manuscripts that they review. Reviewers also have personal experience with the editorial process as published authors.

Several aspects of submitted manuscripts are critiqued by reviewers. First and foremost, papers need to be correct technically. Although only rarely can reviewers know if data presented in manuscripts are indeed accurate, it is possible to assess whether correct experimental procedures or valid computational methods were used to obtain the data. It is also possible for reviewers to assess whether logical reasoning was used by authors to go from data to conclusions.

As with all human endeavors, the quality of peer review depends critically on the skill and dedication of the reviewers. For $\mathrm{JM}^{3}$, we have many reviewers who have been very diligent in their evaluations of published papers, and we should all be grateful for their contributions. Sometimes, a submission may be poorly written. For reviewers, who are usually busy people, reviewing such manuscripts can be very frustrating, because it is difficult to assess technical merit in a manuscript that is written poorly. Nevertheless, most reviewers submit very professional commentary that objectively describes deficiencies in the manuscript, which provides the basis for acceptance, revision, or rejection.

Unfortunately, I have noticed an increasing tendency for reviewers to shift from objective assessment to subjective commentary. All too often, reviewers include unnecessarily caustic and sarcastic comments, representing a notable coarsening of the publication process. While SPIE staff have procedures for filtering reviews so authors do not see such comments, this does require extra effort on the part of staff and delays the publication of quality papers.

While most authors and readers do not see derisive and mocking reviews, many of us have witnessed the coarsening of our intellectual culture during question-and-answer sessions at our conferences. All too frequently, questions are posed in a sarcastic or ridiculing manner. For a presenter at a conference or an author of a paper, it is embarrassing enough to have one's mistakes pointed out; ridicule is unnecessary as well as being unprofessional.

For manuscript reviews, there is a degree of anonymity, in that the names of reviewers are not provided to authors, and it seems that this anonymity has contributed to a greater level of sarcasm and derision in reviews. I think that our technical community would be well-served if reviewers would limit their commentary to objective criticism, even if derisive comments are seen by few people. Indeed, the best reviews are typically very factual.

I am often very impressed by the expertise evinced by our journal's reviewers, who find errors in highly complex arguments that could only be identified by people who are themselves quite knowledgeable and diligent. In the best reviews, these errors are described in fully objective terms, and such reviews are usually welcomed by authors as an aid for improving their papers. It is hoped that all reviewers will view their mission as the improvement of scientific and technical communication in an environment of courteous collegiality.

There are challenges to creating and maintaining such an atmosphere, beginning with the review process itself, which is predicated on the assumption that some papers may be of low

(C) 2021 Society of Photo-Optical Instrumentation Engineers (SPIE) 
quality or incorrect. Good reviewers must necessarily have a skeptical and questioning mindset. Authors and reviewers are viewed from the beginning with a degree of suspicion, being asked to disclose any potential conflicts of interest. Legitimate concerns over conflicts of interest dangerously border the fallacy of association, where validity of an argument is inferred from the author's affiliation, source of funding, or group to which he or she belongs. Everyone involved in the editorial process needs to walk a fine line between justified precautions and invalid forms of argumentation.

The editorial process does not occur in a vacuum. An unfortunate consequence of the rise of social media has been an increase in the use of fallacious forms of argumentation, and not just the fallacy of association. All too frequently, arguments are rejected by attacking the person rather than the argument (argumentum ad hominem) or by claiming that something is incorrect because the majority of people think differently (argumentum consensus gentium).

In the midst of this environment, all of us who participate in the editorial process need to expend effort to avoid the coarsening of the world of lithography and technical publication in general. 